\title{
Significant Gamma Lines from Inert Higgs Dark Matter
}

\author{
Michael Gustafsson $*$ Erik Lundström Lars Bergström \\ Department of Physics, Stockholm University, AlbaNova University Center, SE - 10691 Stockholm, Sweden
}

(Dated: October 4, 2007)

\begin{abstract}
One way to unambiguously confirm the existence of particle dark matter and determine its mass would be to detect its annihilation into monochromatic gamma-rays in upcoming telescopes. One of the most minimal models for dark matter is the inert doublet model, obtained by adding another Higgs doublet with no direct coupling to fermions. For a mass between 40 and $80 \mathrm{GeV}$, the lightest of the new inert Higgs particles can give the correct cosmic abundance of cold dark matter in agreement with current observations. We show that for this scalar dark matter candidate, the annihilation signal of monochromatic $\gamma \gamma$ and $Z \gamma$ final states would be exceptionally strong. The energy range and rates for these gamma-ray line signals make them ideal to search for with the soon upcoming GLAST satellite.
\end{abstract}

PACS numbers: 95.35.+d, 14.80.Cp, 98.70.Rz

Introduction.- - Recent measurements have established that nonbaryonic cold dark matter (CDM) makes up about one quarter of the Universe's total energy budget 1]. Although a variety of candidates have been proposed [2], the nature of this dark matter still remains a mystery.

One particularly attractive class of candidates is that of weakly interacting massive particles (WIMPs), as gauge couplings and masses of the electroweak symmetry breaking scale (i.e. presumably around $100 \mathrm{GeV}$ ) give the right order of magnitude for their thermal relic abundance to explain the dark matter. Moreover, electroweak precision tests indicate that the particle standard model Higgs boson should be light: $m_{h}<144 \mathrm{GeV}$ at $95 \%$ confidence level, with a central value around $76 \mathrm{GeV}[3]$. However, in the standard model, the Higgs boson mass acquires quadratic ultraviolet divergences, requiring finetuning to keep the Higgs mass low if no new divergencecancelling physics appears before the $\mathrm{TeV}$ energy scale (the hierarchy problem). Therefore, new physics can be expected to be found in upcoming high-energy experiments, such as CERN's Large Hadron Collider (LHC).

The most studied scenario which provides both a symmetry to cancel the quadratic divergences in the Higgs sector and a WIMP dark matter candidate is supersymmetry. In the minimal supersymmetric standard model, the lightest Higgs particle is constrained to be lighter than $\sim 135 \mathrm{GeV}$ [4], and although not excluded, some amount of fine-tuning [5] is actually needed to fulfill the experimental lower bound of $114 \mathrm{GeV}[3]$. This has motivated several studies on how to theoretically allow for an increase of the Higgs mass both within supersymmetry and other extensions of the standard model (see, e.g., [5, 6] and references therein).

The Inert Doublet Model (IDM) [6] considered in this Letter is a very minimal extension of the standard model - an added second Higgs doublet $H_{2}$, with an imposed unbroken discrete $Z_{2}$ symmetry that forbids its direct coupling to fermions (i.e. $\mathrm{H}_{2}$ is inert). In the IDM the standard model Higgs mass can be as high as about 500 $\mathrm{GeV}$ and still fulfill experimental precision tests [6]. Fur- thermore, conservation of the $Z_{2}$ parity implies that the lightest inert Higgs particle $\left(H^{0}\right)$ is stable and hence a good dark matter candidate [6, 7]. Although the IDM does not solve the hierarchy problem, but potentially only pushes the need for divergence cancelling physics beyond the reach of upcoming accelerator searches such as the LHC [6, 8], it has the advantage of providing a scalar WIMP dark matter candidate in a very minimalistic way. An electrically neutral $H^{0}$ could therefore be used to represent a larger class of scalar dark matter, similar to how the neutralino in minimal supersymmetry often works as an archetype for supersymmetric dark matter. Actually, the origin of the IDM goes back to the 1970s [9] and has recently received new interest not only because of its potential to allow for a high Higgs mass and a dark matter candidate, but also for generating light neutrinos and leptogenesis (see, e.g., 10] and references therein). This gives strong reasons to study detection prospects for the inert Higgs.

A study of the relic density showed that $H^{0}$ can constitute all dark matter if its mass is roughly $10-80 \mathrm{GeV}$ (or above $500 \mathrm{GeV}$ if parameters are tuned) [11]. Direct detection studies show that the sensitivity of current experiments is far too low, whereas future experiments could cover more of the IDM parameter space 11]. The model can also produce observable signals at the LHC [6]. As shown in [11], indirect detection of the continuous gamma-ray spectrum might be reachable with the upcoming GLAST experiment 12]. However, this study was made for standard model Higgs masses of 120 and $200 \mathrm{GeV}$ which, although giving higher gamma rates, deviates from the motivation for the model of a raised Higgs mass [6] and lacks distinctive detectable features.

An interesting aspect of the IDM is that the $H^{0}$ mass generically has to be below the charged gauge boson mass, since the relatively strong coupling to $W^{+} W^{-}$ would otherwise give a too low relic density to explain the dark matter. Virtual $W$ bosons close to threshold could, on the other hand, significantly enhance loop processes producing monochromatic photons. We show here that 
this is indeed correct and study the dark matter "smoking gun" line signals from the final states $\gamma \gamma$ and, when kinematically allowed, $Z \gamma$. This, in combination with small tree-level annihilation rates into fermions, makes the gamma lines the most promising indirect detection signal.

Calculations of monochromatic gamma lines for both spin-1/2 13] and spin-1 14] dark matter annihilation have been performed earlier. The spin-0 dark matter candidate discussed here has an even more promising gamma line rate.

The Inert Higgs Model and its Constraints. - The IDM framework is an extension of the particle standard model with one additional Higgs doublet $\mathrm{H}_{2}$ and an unbroken $Z_{2}$ symmetry under which $H_{2}$ is odd, $H_{2} \rightarrow-H_{2}$, while all other fields are unchanged (even). The potential for such a model is $[\underline{6}$

$$
\begin{aligned}
& V=\mu_{1}^{2}\left|H_{1}\right|^{2}+\mu_{2}^{2}\left|H_{2}\right|^{2}+\lambda_{1}\left|H_{1}\right|^{4}+\lambda_{2}\left|H_{2}\right|^{4} \\
& +\lambda_{3}\left|H_{1}\right|^{2}\left|H_{2}\right|^{2}+\lambda_{4}\left|H_{1}^{\dagger} H_{2}\right|^{2}+\lambda_{5} \operatorname{Re}\left[\left(H_{1}^{\dagger} H_{2}\right)^{2}\right],
\end{aligned}
$$

where $H_{1}$ is the standard model Higgs doublet, and $\mu_{i}$ and $\lambda_{i}$ are real parameters.

Besides the standard model Higgs particle $(h)$, the physical states derived from the inert doublet $\mathrm{H}_{2}$ are two charged states $\left(H^{ \pm}\right)$and two neutral: one CP-even $\left(H^{0}\right)$ and one CP-odd $\left(A^{0}\right)$, either of which is the dark matter candidate. For definiteness we have chosen $H^{0}$ as the lightest inert particle, although the role of $H^{0}$ and $A^{0}$ can in general be interchanged. The masses are given by

$$
\begin{aligned}
m_{h}^{2} & =-2 \mu_{1}^{2} \\
m_{H^{0}}^{2} & =\mu_{2}^{2}+\left(\lambda_{3}+\lambda_{4}+\lambda_{5}\right) v^{2} \\
m_{A^{0}}^{2} & =\mu_{2}^{2}+\left(\lambda_{3}+\lambda_{4}-\lambda_{5}\right) v^{2} \\
m_{H^{ \pm}}^{2} & =\mu_{2}^{2}+\lambda_{3} v^{2},
\end{aligned}
$$

where $v=m_{h} / \sqrt{4 \lambda_{1}}$ is the vacuum expectation value for $H_{1}$. As usual, the $W$ and $Z$ masses determine $v \sim$ $175 \mathrm{GeV}$. No vacuum expectation value is allowed for $H_{2}$ because of the imposed unbroken $Z_{2}$ parity.

We apply the constraints in [6] concerning vacuum stability, perturbativity, precision tests, accelerator searches and naturalness 24] while using direct detection and charged scalar mass bounds from [11]. We also impose $m_{H^{0}}+m_{A^{0}} \gtrsim m_{Z}$ to not be in conflict with data on the width of the $Z$ boson. Furthermore, in agreement with observations, we constrain the $H^{0}$ abundance to 0.094 $<\Omega_{C D M} h^{2}<0.129$ [1]. The $H^{0}$ relic density calculations have been performed by interfacing the standard numerical packages FormCALC 15] and DARKSUSY [16].

Gamma-ray Lines from Inert Higgs Annihilations.For $H^{0}$ masses below $m_{W}$, only annihilations into fermions lighter than $m_{H^{0}}$ are accessible at tree level. The annihilation rate is given by

$$
v_{\mathrm{rel}} \sigma_{f \bar{f}}=\frac{N_{c} \pi \alpha^{2} m_{f}^{2}}{\sin ^{4} \theta_{W} m_{W}^{4}} \frac{\left(1-\frac{4 m_{f}^{2}}{s}\right)^{3 / 2}\left(m_{H^{0}}^{2}-\mu_{2}^{2}\right)^{2}}{\left(s-m_{h}^{2}\right)^{2}+m_{h}^{2} \Gamma_{h}^{2}},
$$

where $N_{c}$ is a color factor (which equals 1 for leptons and 3 for quarks), $\sqrt{s}$ is the center of mass energy, $\alpha$ the fine-structure constant, $m_{W}$ the $\mathrm{W}$ boson mass, $\theta_{W}$ the weak mixing angle, $\Gamma_{h}$ the decay width of $h$, and $m_{f}$ the final state fermion mass.

The heaviest kinematically allowed fermion state will dominate the tree-level annihilation channels. The contributions to the continuum gamma-ray spectrum from annihilating $H^{0}$ with masses below $m_{W}$ predominantly come from the secondary gamma rays produced in the fragmentation of the bottom quarks, mainly by the sequential production and decay of neutral pions. Because of the harder gamma spectrum from the decay of $\tau$ leptons they contribute significantly at the highest energies, despite their much lower branching ratio. We use [17] to calculate the shape of the continuum spectra.

We now turn to the main issue in this Letter - the very important line signals from direct annihilation of $H^{0}$ pairs into $\gamma \gamma$ and $Z \gamma$. These spectral lines would show up as characteristic dark matter fingerprints at the energies $m_{H^{0}}$ and $m_{H^{0}}-m_{Z}^{2} / 4 m_{H^{0}}$, respectively. The $Z \gamma$ line might not be striclty monochromatic due to the BreitWigner width of the $\mathrm{Z}$ mass, but can still be strongly peaked. When the branching ratio into $Z \gamma$ becomes significant the subsequent decay of the $Z$ boson significantly contributes to the continuum gamma-ray spectrum. The full one-loop Feynman amplitudes were calculated by implementing the IDM into the numerical FormCALC package [15].

Four IDM benchmark models are defined in Table 【. where the two models III and IV are similar to those investigated in [11]. Annihilation rates, branching ratios and relic densities for these models are given in Table As an illustrative example, Fig. 1 shows the predicted gamma spectrum for model I.

The spectral shape with its characteristic peaks in the hitherto unexplored energy range between 30 and 100 $\mathrm{GeV}$ is ideal to search for with the GLAST experiment [18]. In Fig. 2 this is illustrated by showing the predicted fluxes from a $\Delta \Omega=10^{-3}$ sr region around the direction of the galactic center together with existing observations in the same sky direction. For simplicity, we assume a standard Navarro, Frenk and White (NFW) density profile 19] for the dark matter halo in our galaxy $(J \times \Delta \Omega \sim 1$ for $\Delta \Omega=10^{-3}$ sr with the notation of [20]). Processes such as adiabatic compression could enhance the dark matter density significantly near the galactic center (see, e.g., [21]), and we therefore allow our predicted flux to be scaled by a "boost factor".

The Energetic Gamma-Ray Experiment Telescope (EGRET) data, taken from [20], set an upper limit for the continuum part of our spectrum. For example, for benchmark model II one finds that an optimistic, but not necessarily unrealistic [21], boost of $10^{4}$ might be allowed. In such cases, there would be a truly spectacular $\gamma \gamma$ line signal waiting for GLAST. However, to enable 


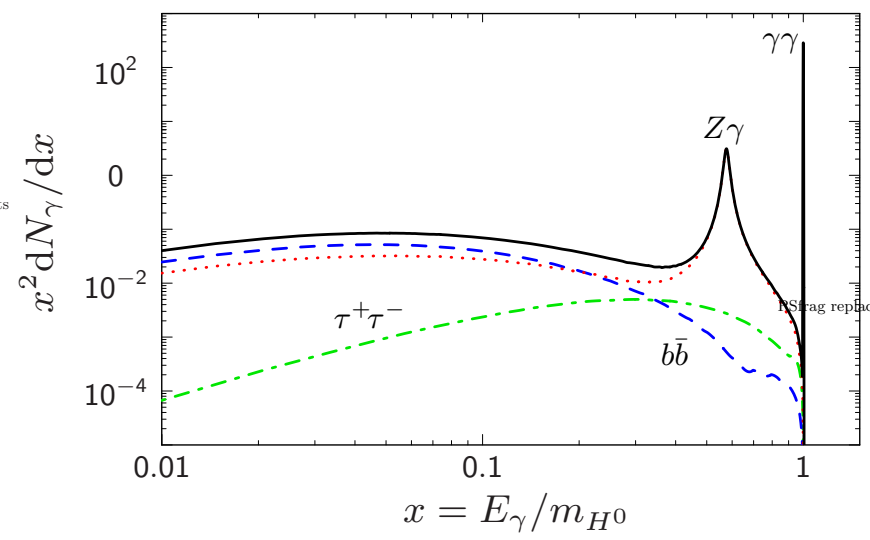

FIG. 1: The total differential photon distribution from annihilations of an inert Higgs dark matter particle (solid line). Shown separately are the contributions from $H^{0} H^{0} \rightarrow b \bar{b}$ (dashed line), $\tau^{+} \tau^{-}$(dash-dotted line) and $Z \gamma$ (dotted line). This is for the benchmark model I in Table $\square$.

detection, boost factors of such magnitudes are not necessary. For $H^{0}$ masses closer to the $W$ threshold the $\gamma \gamma$ annihilation rates become even higher and in addition $Z \gamma$ production becomes important. In fact, these signals would potentially be visible even without any boost at all (especially if the background is low, as might be the case if the EGRET signal is an galactic off-center source as indicated in [22]). Also shown in Fig. 22 is the data from the currently operating air Cherenkov telescope HESS [23]. One may notice that future air Cherenkov telescopes with lower energy thresholds will cover all of the interesting region for this dark matter candidate.

Finally, we have made a systematic parameter scan for $m_{h}=500 \mathrm{GeV}$, calculating the cross section into gamma lines. The previously mentioned constraints allow us to scan the full parameter space for dark matter masses below the $W$ threshold of $80 \mathrm{GeV}$. The dependence on $m_{H^{ \pm}}$and $\lambda_{2}$ is small, and we set these equal to $m_{H^{0}}+120 \mathrm{GeV}$ (to fulfill precision tests) and 0.1, respectively. Importantly, one notes that the right relic density is obtained with a significant amount of early Universe coannihilations with the inert $A^{0}$ particle. The resulting annihilation rates into $\gamma \gamma$ and $Z \gamma$ are shown in Fig. 3, The lower and upper $m_{H^{0}}$ mass bounds come from the accelerator constraints and the effect on the relic density by the opening of the $W^{+} W^{-}$annihilation channel, respectively. For comparison, we show in the same figure

TABLE I: IDM benchmark models. (In units of GeV.)

\begin{tabular}{ccccccc}
\hline \hline Model & $m_{h}$ & $m_{H^{0}}$ & $m_{A^{0}}$ & $m_{H^{ \pm}}$ & $\mu_{2}$ & $\lambda_{2} \times 1 \mathrm{GeV}$ \\
\hline I & 500 & 70 & 76 & 190 & 120 & 0.1 \\
II & 500 & 50 & 58.5 & 170 & 120 & 0.1 \\
III & 200 & 70 & 80 & 120 & 125 & 0.1 \\
IV & 120 & 70 & 80 & 120 & 95 & 0.1 \\
\hline
\end{tabular}

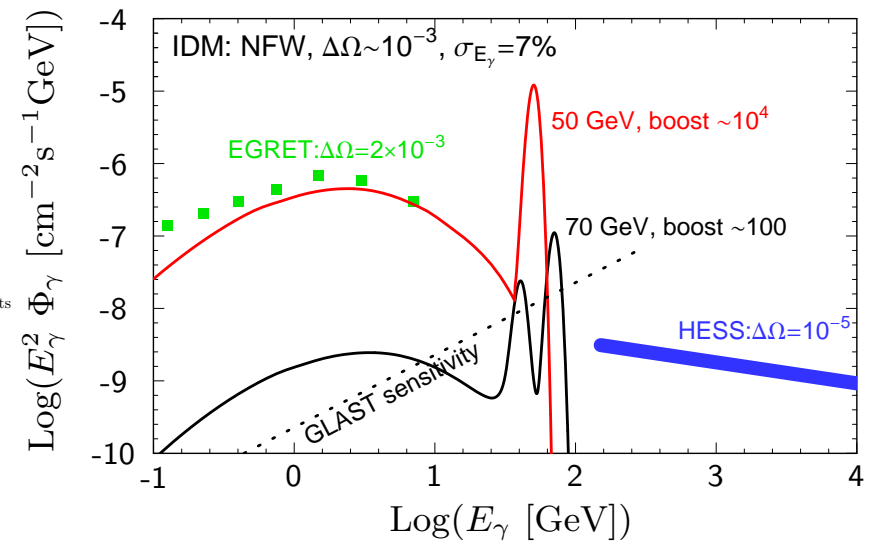

FIG. 2: Predicted gamma-ray spectra from the inert Higgs benchmark models I and II as seen by GLAST (solid lines). The predicted gamma flux is from a $\Delta \Omega=10^{-3} \mathrm{sr}$ region around the direction of the galactic center assuming an $N F W$ halo profile (with boost factors as indicated in the figure) and convolved with a $7 \%$ Gaussian energy resolution. The boxes show EGRET data (which set an upper limit for the continuum signal) and the thick line HESS data in the same sky direction. The GLAST sensitivity (dotted line) is here defined as 10 detected events within an effective exposure of $1 \mathrm{~m}^{2} \mathrm{yr}$ within a relative energy range of $\pm 7 \%$.

the corresponding annihilation rates for the neutralino $(\chi)$ within the minimal supersymmetric standard model. The stronger line signal and smaller spread in the predicted IDM flux are caused by the allowed unsuppressed coupling to $W$ pairs that appear in contributing Feynman loop diagrams.

Summary and Conclusions. - In this Letter, we have investigated the gamma-ray spectrum from the annihilation of the inert Higgs dark matter candidate $H^{0}$. In particular, we have focused on its striking gamma lines which arise at the one-loop level and produce an exceptionally clear dark matter signal.

The gamma line signals are particularly strong for this scalar dark matter model mainly for two reasons: (1) The dark matter mass is just below the kinematic threshold for $W$ production in the zero velocity limit. (2) The dark matter candidate almost decouples from fermions (i.e., couples only via standard model Higgs exchange), while still having ordinary gauge couplings to the gauge bosons. In fact, these two properties could define a more

TABLE II: IDM benchmark model results.

\begin{tabular}{|c|c|c|c|c|c|c|c|}
\hline \multirow[t]{2}{*}{ Model } & \multirow{2}{*}{$\begin{array}{c}v \sigma_{t o t}^{v \rightarrow 0} \\
{\left[\mathrm{~cm}^{3} \mathrm{~s}^{-1}\right]}\end{array}$} & \multicolumn{5}{|c|}{ "Branching ratios [\%]: } & \multirow[t]{2}{*}{$\overline{\Omega_{\mathrm{CDM}} h^{2}}$} \\
\hline & & $\gamma \gamma$ & $Z_{\gamma}$ & $b \bar{b}$ & $c \bar{c}$ & $\tau^{+} \tau^{-}$ & \\
\hline I & $1.6 \times 10^{-28}$ & 36 & 33 & 26 & 2 & 3 & 0.10 \\
\hline II & $8.2 \times 10^{-29}$ & 29 & 0.6 & 60 & 4 & 7 & 0.10 \\
\hline III & $8.7 \times 10^{-27}$ & 2 & 2 & 81 & 5 & 9 & 0.12 \\
\hline IV & $1.9 \times 10^{-26}$ & 0.04 & 0.1 & 85 & 5 & 10 & 0.11 \\
\hline
\end{tabular}




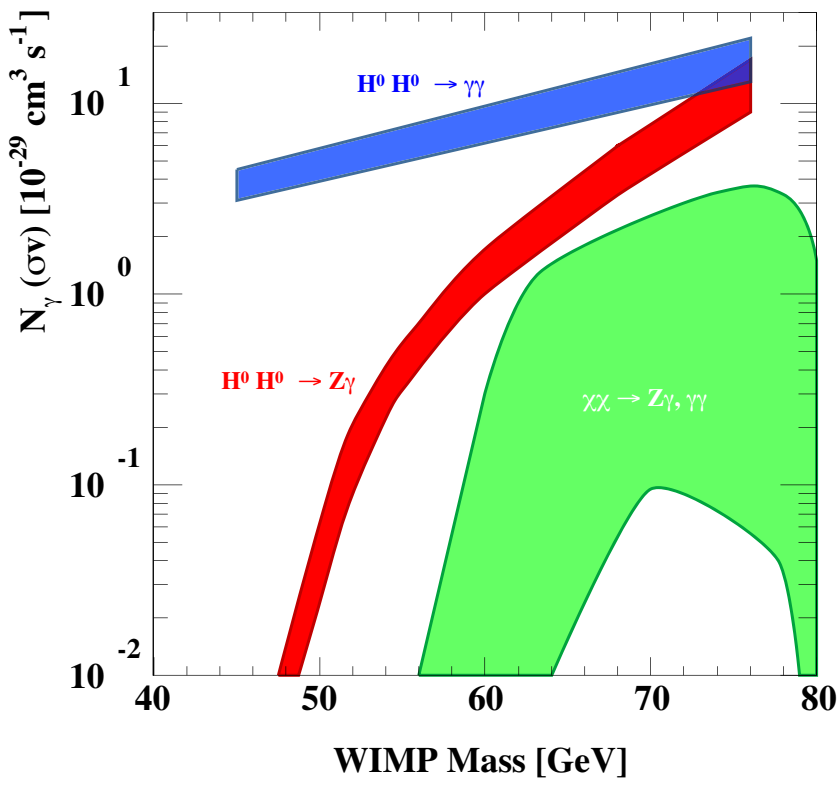

FIG. 3: Annihilation rates into gamma-ray lines $2 v \sigma_{\gamma \gamma}$ (upper band) and $v \sigma_{Z \gamma}$ (middle band) from the scan over the IDM parameter space. For comparison the lower-right region indicates the corresponding results within the minimal supersymmetric standard model as obtained with the DARKSUSY package [16].

general class of models for which the IDM is an attractive archetype. Despite small $H^{0}$ annihilation cross sections, coannihilations with $A^{0}$ in the early Universe can bring the relic density of $H^{0}$ into the correct range. The combination of the low (or even completely vanishing) treelevel annihilation rates today and the strong loop-level processes, due to unsuppressed couplings to virtual $W$ bosons close to threshold, make the gamma lines the far most dominant feature in the resulting gamma spectrum.

Absolute gamma-ray fluxes are, unfortunately, still hard to predict due to the uncertainties in the structure of dark matter halos. It might eventually be the spectral shape that enables a separation of a dark matter signal and the background, in which case a gamma-line would be a striking feature. We have shown that such signals in the IDM are promising features to search for with the GLAST satellite and with future air Cherenkov telescopes. One should bear in mind that the best prospects for detection might not be in the direction of the galactic center, but rather for other sources, such as dwarf galaxies, smaller dark matter clumps or in the extragalactic gamma-ray radiation, where the background is lower.

M.G. thanks Jan Conrad and Alexander Sellerholm for useful discussions on the GLAST performance. M.G. and E.L. thank Thomas Hahn for technical assistance with FormCalC. L.B. and J.E. are grateful to the Swedish Science Research Council (VR) for support.
* Electronic address: michael@physto.se

† Electronic address: erik@physto.se

¥ Electronic address: 1be@physto.se

$\S$ Electronic address: edsjo@physto.se

[1] D. N. Spergel et al., Astrophys. J. Suppl. Ser. 170, 377 (2007) astro-ph/0603449.

[2] G. Bertone, D. Hooper and J. Silk, Phys. Rept. 405, 279 (2005) hep-ph/0404175.

[3] LEP Electroweak Working Group, status of March 2007, http://lepewwg.web.cern.ch/LEPEWWG/.

[4] W. M. Yao et al. (Particle Data Group), J. Phys. G 33, 1 (2006) (http://pdg.lbl.gov)

[5] R. Barbieri, L. J. Hall, Y. Nomura and V. S. Rychkov, Phys. Rev. D 75, 035007 (2007) hep-ph/0607332.

[6] R. Barbieri, L. J. Hall and V. S. Rychkov, Phys. Rev. D 74, 015007 (2006) hep-ph/0603188.

[7] E. Ma, Phys. Rev. D 73, $077301 \quad$ (2006) hep-ph/0601225.

[8] J. A. Casas, J. R. Espinosa and I. Hidalgo, Nucl. Phys. B 777, 226 (2007) hep-ph/0607279.

[9] N. G. Deshpande and E. Ma, Phys. Rev. D 18, 2574 (1978).

[10] E. Ma and U. Sarkar, Phys. Lett. B 653, 288 (2007) arXiv:0705.0074 [hep-ph]].

[11] L. Lopez Honorez, E. Nezri, J. L. Oliver and M. H. G. Tytgat, JCAP 0702, 028 (2007) hep-ph/0612275.

[12] N. Gehrels and P. Michelson [GLAST Collaboration], Astropart. Phys. 11, 277 (1999).

[13] L. Bergström and P. Ullio, Nucl. Phys. B 504, 27 (1997) hep-ph/9706232; P. Ullio and L. Bergstrom, Phys. Rev. D 57, 1962 (1998) hep-ph/9707333; L. Bergström, P. Ullio and J. H. Buckley, Astropart. Phys. 9, 137 (1998) astro-ph/9712318.

[14] L. Bergström, T. Bringmann, M. Eriksson \& M. Gustafsson, JCAP 0504, 004 (2005) hep-ph/0412001.

[15] T. Hahn and M. Perez-Victoria, Comput. Phys. Commun. 118, 153 (1999) hep-ph/9807565; T. Hahn, Comput. Phys. Commun. 140, 418 (2001) hep-ph/0012260.

[16] P. Gondolo, J. Edsjö, P. Ullio, L. Bergström, M. Schelke and E. A. Baltz, JCAP 0407, 008 (2004) astro-ph/0406204.

[17] T. Sjöstrand, S. Mrenna and P. Skands, JHEP 0605, 026 (2006) hep-ph/0603175.

[18] G. Zaharijas and D. Hooper, Phys. Rev. D 73, 103501 (2006) astro-ph/0603540.

[19] J. F. Navarro, C. S. Frenk and S. D. M. White, Astrophys. J. 490, 493 (1997) astro-ph/9611107.

[20] A. Cesarini, F. Fucito, A. Lionetto, A. Morselli and P. Ullio, Astropart. Phys. 21, 267 (2004) astro-ph/0305075.

[21] G. Bertone and D. Merritt, Phys. Rev. D 72, 103502 (2005) astro-ph/0501555.

[22] D. Hooper and B. L. Dingus, Phys. Rev. D 70, 113007 (2004) astro-ph/0210617.

[23] F. Aharonian et al. [H.E.S.S. Collaboration], Phys. Rev. Lett. 97, 221102 (2006) [Erratum-ibid. 97, 249901 (2006)] astro-ph/0610509.

[24] Because of arbitrariness in defining naturalness, we relax this constraint by a factor of 2 compared to [6]. 